Version of Record: https://www.sciencedirect.com/science/article/pii/S1297319X18300617

Manuscript_f2ef1704596adc0f9aec14d11bdae848

\title{
Gut Microbiome and Bone
}

Lidia Ibáñez ${ }^{\mathrm{a}, \mathrm{b}, \mathrm{c}}$, Matthieu Rouleau ${ }^{\mathrm{a}, \mathrm{b}}$, Abdelilah Wakkach ${ }^{\mathrm{a}, \mathrm{b}}$, Claudine Blin-Wakkach ${ }^{\mathrm{a}, \mathrm{b}}$

${ }^{a}$ CNRS UMR7370, LP2M, Faculté de Médecine, 06107 Nice, France

${ }^{\mathrm{b}}$ Université Nice Sophia Antipolis, 06100 Nice, France

${ }^{\mathrm{c}}$ Department of Pharmacy, Cardenal Herrera-CEU University, 46115 Alfara del Patriarca, València, Spain

Corresponding author: Claudine Blin-Wakkach, CNRS UMR7370, LP2M, Faculté de Médecine, 28 avenue de Valombrose, 06107 Nice cedex 2, France

E-mail: blin@unice.fr 


\begin{abstract}
The gut microbiome is now viewed as a tissue that interacts bidirectionally with the gastrointestinal, immune, endocrine, and nervous systems, affecting the cellular responses in numerous organs. Evidence is accumulating of gut microbiome involvement in a growing number of pathophysiological processes, many of which are linked to inflammatory responses. More specifically, data acquired over the last decade point to effects of the gut microbiome on bone mass regulation and on the development of bone diseases (such as osteoporosis) and of inflammatory joint diseases characterized by bone loss. Mice lacking a gut microbiome have bone mass abnormalities that can be reversed by gut recolonization. Changes in gut microbiome composition have been reported in mice with estrogendeficiency osteoporosis and have also been found in a few studies in humans. Probiotic therapy decreases bone loss in estrogen-deficient animals. The effect of the gut microbiome on bone tissue involves complex mechanisms including modulation of $\mathrm{CD}^{+} \mathrm{T}$-cell activation, control of osteoclastogenic cytokine production, and modifications in hormone levels. This complexity may contribute to explain the discrepancies across study results depending on age, gender, genetic background, and treatment duration. Further elucidation of the mechanisms involved is needed. However, the available data hold promise that gut microbiome manipulation may prove of interest in the management of bone diseases.
\end{abstract}

Keywords: Osteoclasts. Osteoporosis. Osteoimmunology. T lymphocytes. Gut microbiome. 


\section{1- Introduction}

The $10^{14}$ microorganisms in the gut microbiome (GM) continuously engage in a dynamic dialog with the host cells [1]. The GM is now viewed as an organ that contributes to digestive function, transforming complex food components such as fibers and carbohydrates into absorbable nutrients including short-chain fatty acids [2]. Commensal bacteria produce factors that modulate the host immune responses, help preserve gut barrier integrity, defend against pathogenic microorganisms, and contribute to immune system development and regulation. These effects are particularly important for the mucosal immune system in the gut, which maintains tolerance toward food allergens and the GM while ensuring protection against pathogenic microorganisms. Thus, the GM regulates the development and function of lymphoid cells; the polarization of gut T cells, most notably Th17 cells; and the production of cytokines $[3,4]$.

The composition of the GM can vary over the life span depending on age, genetic factors, diet, medication intake, and host immune status. The interactions of the GM with the host may be altered by dysbiosis, which is defined as adverse changes in bacterial composition, diversity, and function. When dysbiosis occurs, the GM loses its protective capabilities, the gut barrier is impaired, and the host fails to effectively control the dissemination of GM components into the tissues. The resulting stimulation of the immune system can lead to a variety of diseases. Dysbiosis is associated not only with Crohn's disease, irritable bowel syndrome, and celiac disease; but also with metabolic, cardiovascular, and neurodegenerative diseases, as well as with inflammatory joint disease [5-7]. In recent years, the effect of the GM on bone tissue has been evaluated in germ-free animals lacking a GM, animals given antibiotics or probiotics to modify the GM, and 
humans. Although the study results are somewhat conflicting, they establish the GM as a major regulator of bone mineral density (BMD), chiefly via effects on the immune system.

\section{2- Interactions of bone and immune system -- bone resorption}

The immune system is central to BMD control under abnormal conditions. The receptor activator of NFKB ligand (RANKL) is the main cytokine involved in osteoclast differentiation. RANKL is produced by mesenchymal cells, osteoblasts, and osteocytes in the bone marrow. During inflammation, activated $\mathrm{CD}^{+} \mathrm{T}$ cells are also a source of RANKL [8,9], as well as of other cytokines including interleukin (IL)-17 and tumor necrosis factor alpha (TNF- $\alpha$ ), which stimulate osteoclastogenesis $[10,11]$. Changes in T-cell activation levels therefore affect osteoclast differentiation [12,13]. Among helper CD4 ${ }^{+} \mathrm{T}$-cell subsets, only Th17 cells have been found to promote osteoclastogenesis in vitro [14]. In vivo, Th17 cells are associated with enhanced osteoclast differentiation in mice and humans during inflammatory states $[13,15,16]$. Crosstalk between Th17 cells and osteoclasts has also been demonstrated in Crohn's disease in both mice and humans [16,17]. Th17 cells activated at sites of bowel inflammation produce high levels of the osteoclastogenic factors RANKL, IL17, and TNF- $\alpha$ [17]. They migrate to the bone marrow, where they strongly enhance osteoclast differentiation by producing cytokines and upregulating RANKL expression by mesenchymal cells [17]. Th17 cells also upregulate the expression by mesenchymal cells of chemokines (MCP1, MIP1 $\alpha$ ) that attract the monocytic precursors of osteoclasts, increasing their recruitment to the bone marrow [17]. The peripheral blood of patients with Crohn's disease contains Th17 cells that exhibit the same osteoclastogenic properties and therefore probably contribute to the bone loss often seen in this disease [16,17]. Th17 cells activated in the gut are thus crucially involved in inflammatory bone loss destruction. 
Interestingly, mice lacking a GM have no Th17 cells in their gut wall. Introducing certain bacterial species into the gut efficiently induces Th17 cell differentiation, demonstrating the importance of the GM in the emergence of these cells $[3,4]$. Th17 cells are characterized by considerable phenotypic and functional plasticity [18]. They not only protect against pathogenic bacteria [19] but also contribute to many chronic inflammatory diseases, some of which have bone loss among their features, such as Crohn's disease [20], inflammatory joint disease [21], spondyloarthritis [22], and psoriasis [23]. These data lend credence to the existence of a link between the GM and bone resorption via the emergence of Th17 cells.

\section{3- Gut microbiome (gm) and bone formation}

Several clinical studies reported about two decades ago pointed to an association between excessive bacterial proliferation in the gut and decreased BMD [24]. Patients with small intestinal bacterial overgrowth syndrome had low BMD values and osteomalacia, and some of them had high levels of the proinflammatory cytokines TNF- $\alpha$ and IL-1, as well as increased osteoclast activation. Another feature of this syndrome is the development of nutritional deficiencies due to nutrient consumption by the gut bacteria. Thus, insufficient intestinal absorption of calcium, phosphate, and vitamin D contribute to the bone manifestations of the syndrome [24]. Consequently, these studies cannot provide evidence of a direct link between the GM and the bone phenotype.

Convincing evidence of GM involvement in bone tissue development was obtained only a decade later, via studies of germ-free mice, which have no GM. Given the effect of the GM on metabolism, absence of a GM from birth induces a host of physiological and 
metabolic alterations. The absorption of calories, vitamins, and nutrients is decreased; height and weight gains are delayed, and many organs are abnormally small [25]. Another consequence is immaturity of the immune, vascular, endocrine, intestinal, and nervous systems, all of which are involved in regulating bone mass (Figure 1) [26,27].

Female C57Bl/6J mice lacking a GM from birth have higher BMD values in both trabecular and cortical bone compared to their conventionally reared counterparts [28]. These high BMD values reflect decreases in the numbers of osteoclast precursors and mature osteoclasts, which translate into diminished bone resorption. Bone formation is unchanged. Additional contributors to the reduction in bone resorption include decreases in $\mathrm{CD}^{+}{ }^{+} \mathrm{T}$-cell frequency and in the expression levels of TNF- $\alpha$ and IL-6 in bone marrow [28].

Colonization of germ-free mice with GM from conventionally raised mice corrects the BMD and immune parameter values, confirming that the GM controls osteoclast differentiation during skeletal growth [28]. Among the receptors involved in recognition of bacteria by the immune system, the nucleotide-binding oligomerization domain proteins NOD1 and NOD2 bind bacterial peptidoglycans and activate the NFKB pathway. In knockout mice for both NOD1 and NOD2, changes in the GM have no effect on BMD or on TNF- $\alpha$ and RANKL expression, demonstrating that NOD1 and NOD2 play a critical role in GM effects on bone [29].

However, GM effects on bone development are more complex than appears to the casual eye. A study in germ-free juvenile male Balb/c mice reported in 2016 produced contradictory results, with delays in most of the main growth parameters and shorter femurs compared to conventionally raised controls [30]. The discrepancy between the results of the two studies may be ascribable to differences in the genetic background, gender, or age of the mice. The C57B1/6J and Balb/C strains differ regarding GM diversity, IgA levels (which modulate GM diversity), and the T-cell profile [31,32]. Treatment duration is also of the 
utmost importance. Colonization of germ-free mice by GM from normal mice is followed by increases in both bone resorption and bone formation. The bone resorption enhancement, however, is transient, resolving after 4 weeks. Thus, the GM exerts an acute catabolic effect on bone tissue [33]. In the longer term, bone formation predominates, leading to increased skeletal growth and bone mass correction, indicating a long-term anabolic effect of the GM on bone tissue [33]. This anabolic effect coincides with an increase in the production of insulin growth factor (IGF)-1. IGF-1 levels in serum and bone marrow are low in germ-free mice and in mice given antibiotics. They increase after gut recolonization or ingestion of a diet high in short-chain fatty acids, which are produced when GM bacteria break down polysaccharides [33]. Given the major role for IGF1 in skeletal development [34], control of IGF1 production is among the mechanisms that may explain the effect of the GM on bone growth.

Another major issue is the influence of gender. Male and female C57B1/6J mice with significant GM alterations induced by low-dose penicillin treatment started at birth exhibit different phenotypes [35]. The females, but not the males, have higher adulthood BMD values compared to untreated animals [35]. In addition, the BMD increase seen after gut recolonization of germ-free mice is more marked in the long-term in males than in females [33]. Interestingly, the GM in male and female mice is similar before puberty but differs in adulthood, and the differences contribute to increase the susceptibility to certain diseases, such as type I diabetes in females [36]. Gender-related differences in the GM have also been reported in humans $[37,38]$. Thus, two-way interactions between the GM and sex hormones influence the composition of the GM and the response of host tissues, including bone, to the GM [39].

\section{4- Gut microbiome and bone destruction}


Potential effects of the GM on bone mass during estrogen-deficient osteoporosis have been investigated. At the menopause, the drop in estrogen production leads to a decline in bone mass due to a combination of diminished bone formation and increased bone resorption. In mice with osteoporosis induced by ovariectomy, osteoclast activity is enhanced due to increased production of RANKL and TNF- $\alpha$ in the bone marrow [40]. The production of these two factors is mediated by $\mathrm{CD}^{+} \mathrm{T}$ cells [40]. Thus, mice deficient in $\mathrm{CD}^{+} \mathrm{T}$ cells do not lose bone after being ovariectomized [41]. Similarly, compared to premenopausal women and to postmenopausal women without osteoporosis, patients with postmenopausal osteoporosis exhibit RANKL and TNF- $\alpha$ overproduction by peripheral blood $\mathrm{CD}^{+} \mathrm{T}$ cells [42].

The central role for the GM in controlling lymphocyte activation [4] and its two-way interactions with sex hormones [36] have prompted studies in mice designed to assess the effect of the GM on the BMD decrease induced by estrogen deprivation. In several studies of ovariectomized mice, a protective effect against bone loss was obtained by the administration of probiotics such as Lactobacillus (L) reuteri, L. paracasei, L. plantarum, Bifidobacterium (B) longum, and mixtures of several species (B. breve, B. longum, $B$. infantis, L. acidophilus, L. plantarum, L. paracasei, L. bulgaricus, and Streptococcus thermophilus) [43-45]. This finding was confirmed in a model of osteoporosis induced by the gonadotropin-releasing hormone agonist leuprolide, which suppresses the production of sex hormones, including estrogens, in the long term. The estrogen deficiency led to bone loss in conventionally raised mice but not in germ-free mice [46]. In conventionally raised mice, estrogen deficiency increased gut wall permeability and the proportion of $\mathrm{CD}^{+} \mathrm{T}$ cells producing RANKL, IL-17, and TNF- $\alpha$, thereby enhancing osteoclastogenesis (Figure 2) [46]. Germ-free mice exhibited none of these modifications [46]. In a small preliminary 
study of normal controls, patients with osteopenia, and patients with osteoporosis (6 per group), differences in the GM were found across the three groups. This finding requires confirmation in a larger sample size but suggests a role for dysbiosis in human osteoporosis [47].

GM alterations have a major impact in inflammatory joint disease accompanied by bone loss. Mice and rats whose GM is lacking or altered by antibiotics have decreased susceptibility to arthritis and spondyloarthritis [48,21]. Gut dysbiosis has been reported in patients with rheumatoid arthritis or spondyloarthritis [49-51]. In addition, in rats with collagen-induced arthritis, the administration of probiotics protects against inflammation and bone loss [52]. Thus, the effect of the GM on bone is not confined to conditions characterized by hormonal changes and can involve a wide variety of mechanisms.

Recent data indicate that osteoporosis and inflammatory joint diseases share a common immune component. The gut barrier is a crucial player in host-GM interactions. Dysbiosis is associated with gut barrier alterations that promote the dissemination of bacteria and of the factors they produce. The gut barrier is altered in both inflammatory joint disease and estrogen deficiency $[46,53,54]$. In both situations, the alterations are accompanied with enhanced $\mathrm{CD}^{+}{ }^{+} \mathrm{T}$-cell activation and increased production of the proinflammatory and osteoclastogenic cytokines IL-17, TNF- $\alpha$, IL1 $\beta$ and RANKL [44-46].

By modulating the gut immune response, dysbiosis also alters monocyte and lymphocyte migration to tissues, including the bone marrow. In germ-free mice, the numbers of monocytes and osteoclastic precursors in the bone marrow are diminished but return to normal after gut colonization with GM from control mice $[28,55]$. In addition, changes in the GM translate into alterations in monocyte trafficking [56]. In patients with Crohn's disease, severe bone loss, and dysbiosis, the Th17 cells can migrate to the bone marrow and induce the recruitment of osteoclastic precursors, thereby prompting a massive increase in 
osteoclastogenesis [17]. Whereas osteoclasts from normal mice induce the generation of regulatory $\mathrm{T}$ cells (Tregs), osteoclasts generated during inflammatory states activate $\mathrm{CD}^{+} \mathrm{T}$ cells that produce TNF- $\alpha$ [57]. This difference is ascribable to the cellular origin of the osteoclasts and reflects differences in osteoclastic precursor recruitment under normal conditions versus during dysbiosis-related inflammatory states.

\section{5- Future directions}

A growing number of studies indicate a major role for the GM in regulating bone mass both during growth and during disease states. The GM can act in combination with other factors including the diet, genetic susceptibility, lifestyle, and medications. Another mechanism of action of the GM is a direct effect due to the dissemination of bacteria [58] or of the factors they produce, which then activate the inflammatory responses in various tissues including the bone marrow However, the mechanisms involved are complex, and further investigations are needed to clarify and to control them.

Restoring a balanced GM is now being considered as a therapeutic tool for various diseases. Methods of modifying the GM include dietary changes and supplementation with probiotics such as short-chain fatty acids, oligosaccharides, carbohydrates, and dietary fiber. These supplements are metabolized by certain bacterial strains, whose growth they promote, thereby modifying the composition of the GM. The modified GM stimulates antiinflammatory responses and promotes the intestinal absorption of calcium, thereby increasing $\mathrm{BMD}$, an effect demonstrated in mice given short-chain fatty acids by gavage [33]. Oligosaccharides from human milk have shown similar beneficial effects on bone [59]. Probiotics also have a substantial effect on BMD. Various strains of Lactobacillus and Bifidobacterium have antiinflammatory effects, enhance vitamin D abruption, and diminish 
osteoclast differentiation, thereby protecting against the bone mass loss induced by ovariectomy in mice [43-45]. The effects of probiotics in humans are being evaluated in several clinical trials registered on ClinicalTrials.gov, but the results are not yet available.

Another approach is GM transplantation, which has been widely used in mice to demonstrate that the GM was involved in many disease states, including those affecting the bone $[28,33,46]$. In humans, GM transplantation has been used successfully to treat bowel diseases such as colitis due to antibiotic-resistant bacteria [60]. Clinical studies are under way to evaluate the efficacy of GM transplantation in rheumatic diseases. However, no effects on bone have been reported. Interest is growing in GM manipulation as a therapeutic tool, and additional research is therefore needed to elucidate the mechanisms involved and to evaluate the efficacy of this approach in bone diseases. The beneficial effects of GM manipulation in preclinical models suggest promise for the treatment of bone disease.

\section{Acknowledgments}

The work conducted by our group is supported by the French National Research Agency (ANR, ANR-16-CE14-0030-01), Biocodex, and the Fondation pour la Recherche Médicale (FRM, ECO20160736019). The figures accompanying this article were created using illustrations from Servier Medical Art (http://smart.servier.com).

\section{Disclosure of interest}

CBW has received a research grant from Biocodex. 


\section{6- References}

[1] Marchesi J, Shanahan F. The normal intestinal microbiota: Curr Opin Infect Dis 2007;20:508-13.

[2] Topping DL, Clifton PM. Short-chain fatty acids and human colonic function: roles of resistant starch and nonstarch polysaccharides. Physiol Rev 2001;81:1031-64.

[3] Ivanov II, Frutos R de L, Manel N, et al. Specific microbiota direct the differentiation of IL-17-producing T-helper cells in the mucosa of the small intestine. Cell Host Microbe $2008 ; 4: 337-49$.

[4] Gaboriau-Routhiau V, Rakotobe S, Lécuyer E, et al. The key role of segmented filamentous bacteria in the coordinated maturation of gut helper T cell responses. Immunity 2009;31:677-89.

[5] Hand TW, Vujkovic-Cvijin I, Ridaura VK, Belkaid Y. Linking the microbiota, chronic disease, and the immune system. Trends Endocrinol Metab TEM 2016;27:831-43. [6] Van de Wiele T, Van Praet JT, Marzorati M, Drennan MB, Elewaut D. How the microbiota shapes rheumatic diseases. Nat Rev Rheumatol 2016;12:398-411.

[7] Ni J, Wu GD, Albenberg L, Tomov VT. Gut microbiota and IBD: causation or correlation? Nat Rev Gastroenterol Hepatol 2017;14:nrgastro.2017.88.

[8] Kong YY, Yoshida H, Sarosi I, et al. OPGL is a key regulator of osteoclastogenesis, lymphocyte development and lymph-node organogenesis. Nature 1999;397:315-23.

[9] Nakashima T, Hayashi M, Fukunaga T, et al. Evidence for osteocyte regulation of bone homeostasis through RANKL expression. Nat Med 2011;17:1231-4.

[10] Kotake S, Udagawa N, Takahashi N, et al. IL-17 in synovial fluids from patients with rheumatoid arthritis is a potent stimulator of osteoclastogenesis. J Clin Invest 1999;103:1345-52.

[11] Lam J, Takeshita S, Barker JE, Kanagawa O, Ross FP, Teitelbaum SL. TNF-alpha 
induces osteoclastogenesis by direct stimulation of macrophages exposed to permissive levels of RANK ligand. J Clin Invest 2000;106:1481-8.

[12] Kong Y-Y, Feige U, Sarosi I, et al. Activated T cells regulate bone loss and joint destruction in adjuvant arthritis through osteoprotegerin ligand. Nature 1999;402:304-9.

[13] Wakkach A, Mansour A, Dacquin R, et al. Bone marrow microenvironment controls the in vivo differentiation of murine dendritic cells into osteoclasts. Blood 2008;112:507483.

[14] Sato K, Suematsu A, Okamoto K, et al. Th17 functions as an osteoclastogenic helper T cell subset that links T cell activation and bone destruction. J Exp Med 2006;203:2673-82. [15] Pöllinger B, Junt T, Metzler B, et al. Th17 cells, not IL-17+ $\gamma \delta$ T cells, drive arthritic bone destruction in mice and humans. J Immunol Baltim Md 1950 2011;186:2602-12.

[16] Oostlander AE, Everts V, Schoenmaker T, et al. T cell-mediated increased osteoclast formation from peripheral blood as a mechanism for Crohn's disease-associated bone loss. J Cell Biochem 2012;113:260-8.

[17] Ciucci T, Ibáñez L, Boucoiran A, et al. Bone marrow Th17 TNF $\alpha$ cells induce osteoclast differentiation, and link bone destruction to IBD. Gut 2015;64:1072-81.

[18] Peters A, Lee Y, Kuchroo VK. The many faces of Th17 cells. Curr Opin Immunol 2011;23:702-6.

[19] Ivanov II, Atarashi K, Manel N, et al. Induction of intestinal Th17 cells by segmented filamentous bacteria. Cell 2009;139:485-98.

[20] Khor B, Gardet A, Xavier RJ. Genetics and pathogenesis of inflammatory bowel disease. Nature 2011;474:307-17.

[21] Wu H-J, Ivanov II, Darce J, et al. Gut-residing segmented filamentous bacteria drive autoimmune arthritis via T helper 17 cells. Immunity 2010;32:815-27.

[22] Glatigny S, Fert I, Blaton MA, et al. Proinflammatory Th17 cells are expanded and 
induced by dendritic cells in spondylarthritis-prone HLA-B27-transgenic rats. Arthritis Rheum 2012;64:110-20.

[23] Nograles KE, Zaba LC, Guttman E, et al. Th17 cytokines interleukin (IL)-17 and IL22 modulate distinct inflammatory and keratinocyte-response pathways. Br J Dermatol 2008;159:1092-102.

[24] Stotzer P-O, Johansson C, Mellström D, Lindstedt G, Kilander AF. Bone mineral density in patients with small intestinal bacterial overgrowth. Hepatogastroenterology 2003;50:1415-8.

[25] Wallace JG, Gohir W, Sloboda DM. The impact of early life gut colonization on metabolic and obesogenic outcomes: what have animal models shown us? J Dev Orig Health Dis 2016;7:15-24.

[26] Clarke G, Stilling RM, Kennedy PJ, Stanton C, Cryan JF, Dinan TG. Minireview: Gut microbiota: the neglected endocrine organ. Mol Endocrinol Baltim Md 2014;28:122138.

[27] Kennedy PJ, Cryan JF, Dinan TG, Clarke G. Kynurenine pathway metabolism and the microbiota-gut-brain axis. Neuropharmacology 2017;112:399-412.

[28] Sjögren K, Engdahl C, Henning P, et al. The gut microbiota regulates bone mass in mice. J Bone Miner Res Off J Am Soc Bone Miner Res 2012;27:1357-67.

[29] Ohlsson C, Nigro G, Boneca IG, Bäckhed F, Sansonetti P, Sjögren K. Regulation of bone mass by the gut microbiota is dependent on NOD1 and NOD2 signaling. Cell Immunol 2017;317:55-8.

[30] Schwarzer M, Makki K, Storelli G, et al. Lactobacillus plantarum strain maintains growth of infant mice during chronic undernutrition. Science 2016;351:854-7.

[31] Fransen F, Zagato E, Mazzini E, et al. BALB/c and C57BL/6 mice differ in polyreactive iga abundance, which impacts the generation of antigen-specific $\operatorname{IgA}$ and 
microbiota diversity. Immunity 2015;43:527-40.

[32] Stanisavljević S, Đedović N, Vujičić M, et al. Strain-specific helper T cell profile in the gut-associated lymphoid tissue. Immunol Lett 2017;190:282-8.

[33] Yan J, Herzog JW, Tsang K, et al. Gut microbiota induce IGF-1 and promote bone formation and growth. Proc Natl Acad Sci U S A 2016;113:E7554-63.

[34] Yakar S, Courtland H-W, Clemmons D. IGF-1 and bone: New discoveries from mouse models. J Bone Miner Res Off J Am Soc Bone Miner Res 2010;25:2543-52.

[35] Cox LM, Yamanishi S, Sohn J, et al. Altering the intestinal microbiota during a critical developmental window has lasting metabolic consequences. Cell 2014;158:705. [36] Yurkovetskiy L, Burrows M, Khan AA, et al. Gender bias in autoimmunity is influenced by microbiota. Immunity 2013;39:400-12.

[37] Mueller S, Saunier K, Hanisch C, et al. Differences in fecal microbiota in different European study populations in relation to age, gender, and country: a cross-sectional study. Appl Environ Microbiol 2006;72:1027-33.

[38] Dominianni C, Sinha R, Goedert JJ, et al. Sex, body mass index, and dietary fiber intake influence the human gut microbiome. PloS One 2015;10:e0124599.

[39] Baker JM, Al-Nakkash L, Herbst-Kralovetz MM. Estrogen-gut microbiome axis: Physiological and clinical implications. Maturitas 2017;103:45-53.

[40] Roggia C, Gao Y, Cenci S, et al. Up-regulation of TNF-producing T cells in the bone marrow: A key mechanism by which estrogen deficiency induces bone loss in vivo. Proc Natl Acad Sci 2001;98:13960-5.

[41] Cenci S, Weitzmann MN, Roggia C, et al. Estrogen deficiency induces bone loss by enhancing T-cell production of TNF- $\alpha$. J Clin Invest 2000;106:1229-37.

[42] D’Amelio P, Grimaldi A, Di Bella S, et al. Estrogen deficiency increases osteoclastogenesis up-regulating T cells activity: a key mechanism in osteoporosis. Bone 
2008;43:92-100.

[43] Parvaneh K, Ebrahimi M, Sabran MR, et al. Probiotics (Bifidobacterium longum) increase bone mass density and upregulate Sparc and Bmp-2 Genes in rats with bone loss resulting from ovariectomy. BioMed Res Int 2015;2015:1-10.

[44] Britton RA, Irwin R, Quach D, et al. Probiotic L. reuteri treatment prevents bone loss in a menopausal ovariectomized mouse model. J Cell Physiol 2014;229:1822-30.

[45] Ohlsson C, Engdahl C, Fåk F, et al. Probiotics protect mice from ovariectomyinduced cortical bone loss. PloS One 2014;9:e92368.

[46] Li J-Y, Chassaing B, Tyagi AM, et al. Sex steroid deficiency-associated bone loss is microbiota dependent and prevented by probiotics. J Clin Invest 2016;126:2049-63.

[47] Wang J, Wang Y, Gao W, et al. Diversity analysis of gut microbiota in osteoporosis and osteopenia patients. PeerJ 2017;5:e3450.

[48] Taurog JD, Richardson JA, Croft JT, et al. The germfree state prevents development of gut and joint inflammatory disease in HLA-B27 transgenic rats. J Exp Med 1994;180:2359-64.

[49] Vaahtovuo J, Munukka E, Korkeamäki M, Luukkainen R, Toivanen P. Fecal microbiota in early rheumatoid arthritis. J Rheumatol 2008;35:1500-5.

[50] Zhang X, Zhang D, Jia H, et al. The oral and gut microbiomes are perturbed in rheumatoid arthritis and partly normalized after treatment. Nat Med 2015;21:895-905.

[51] Breban M, Tap J, Leboime A, et al. Faecal microbiota study reveals specific dysbiosis in spondyloarthritis. Ann Rheum Dis 2017;76:1614-22.

[52] Amdekar S, Singh V, Singh R, Sharma P, Keshav P, Kumar A. Lactobacillus casei reduces the inflammatory joint damage associated with collagen-induced arthritis (CIA) by reducing the pro-inflammatory cytokines: Lactobacillus casei: COX-2 inhibitor. J Clin Immunol 2011;31:147-54. 
[53] Kerr SW, Wolyniec WW, Filipovic Z, et al. Repeated measurement of intestinal permeability as an assessment of colitis severity in HLA-B27 transgenic rats. J Pharmacol Exp Ther 1999;291:903-10.

[54] Martínez-González O, Cantero-Hinojosa J, Paule-Sastre P, Gómez-Magán JC, Salvatierra-Ríos D. Intestinal permeability in patients with ankylosing spondylitis and their healthy relatives. Br J Rheumatol 1994;33:644-7.

[55] Khosravi A, Yáñez A, Price JG, et al. Gut microbiota promote hematopoiesis to control bacterial infection. Cell Host Microbe 2014;15:374-81.

[56] Bain CC, Bravo-Blas A, Scott CL, et al. Constant replenishment from circulating monocytes maintains the macrophage pool in the intestine of adult mice. Nat Immunol 2014;15:929-37.

[57] Ibáñez L, Abou-Ezzi G, Ciucci T, et al. Inflammatory osteoclasts prime TNF $\alpha$ producing CD4(+) T cells and express CX3 CR1. J Bone Miner Res 2016;31:1899-1908. [58] Burcelin R, Serino M, Chabo C, et al. Metagenome and metabolism: the tissue microbiota hypothesis. Diabetes Obes Metab 2013;15:61-70.

[59] Charbonneau MR, O’Donnell D, Blanton LV, et al. Sialylated milk oligosaccharides promote microbiota-dependent growth in models of infant undernutrition. Cell 2016;164:859-71.

[60] Cammarota G, Ianiro G, Gasbarrini A. Fecal Microbiota Transplantation for the treatment of Clostridium difficile infection: A systematic review. J Clin Gastroenterol 2014;48:693-702. 
FIGURE LEGENDS

\section{Figure 1: Link between the gut microbiome and bone}

The gut microbiome contributes to preserve gut barrier integrity and digestive system maturity. GM alterations lead to increased dissemination of bacteria-derived compounds and to changes in the expression of cytokines and growth factors. As a result, impairments occur in the responses of the immune, endocrine, vascular, and nervous systems, all of which contribute to regulate bone cell differentiation and/or function.

\section{Figure 2: Gut microbiome alterations and osteoclast differentiation}

Alterations in the gut microbiome result in Th17 cell stimulation and in increased production of the osteoclastogenic cytokines TNF- $\alpha$, IL-17, and RANKL in the gut. Th17 cells migrate to the bone marrow, where increases in the same cytokines enhance the differentiation of osteoclasts, thereby diminishing bone mineral density (OCLs, osteoclasts) 


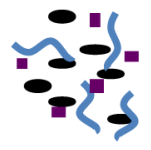

Microbiome

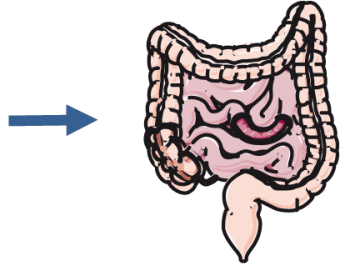

Digestive system
Cytokines, growth factors, bacterial factors
Immune

\section{system}

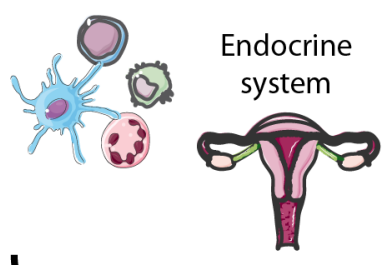

Nervous

system

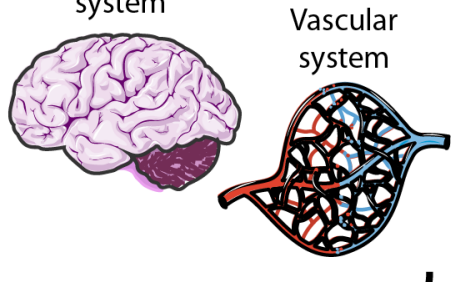

Hormones, neuromediators, cytokines, growth factors
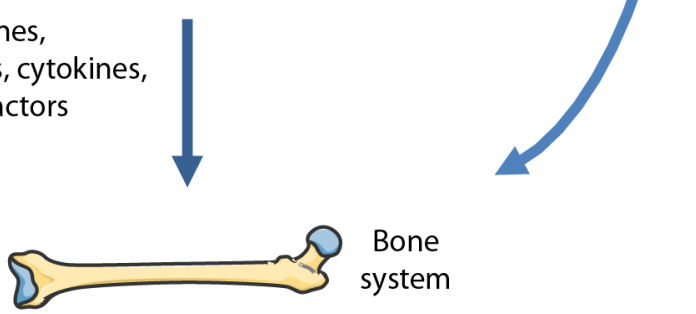

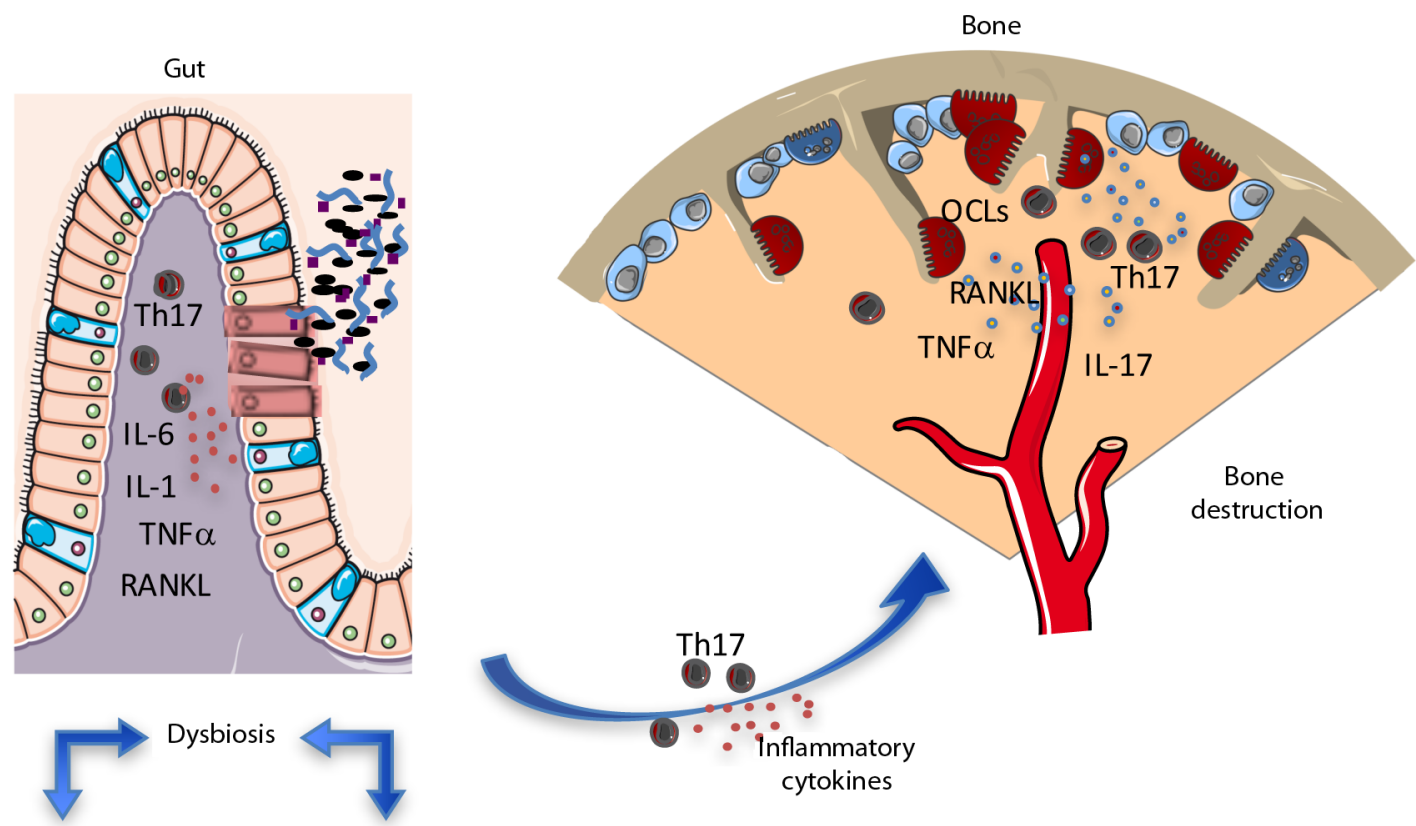

Proinflammatory

cytokines

\section{Dysbiosis}

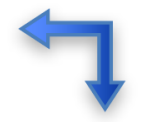

Gut barrier alterations 